\title{
WHAT DETERMINES NEARSHORE SANDBAR RESPONSE?
}

\author{
Marije Smit ${ }^{1}$, Ad Reniers ${ }^{2}$, Marcel Stive ${ }^{3}$
}

\begin{abstract}
Nearshore sandbars appear with various patterns which may change over time. From observations, these changes seem to be related to changes in hydrodynamic conditions, although observed length scales could not be related directly to occurring wave conditions. The current work investigated the role of both the concurrent and previous hydrodynamics as well as the role of the pre-existing morphological variability of a nearshore bar system. A suite of modeling efforts using a depth-averaged process-based model was analysed on predicted length scales, response times and evolving levels of variability. It was found that with small or moderate hydrodynamic forcing, an existing pattern would remain. Only when the existing pattern was alongshore uniform, the bar pattern would change in response to the conditions. When the hydrodynamic conditions are extreme, an existing pattern can be erased, resulting in an alongshore uniform bathymetry - a reset-event.
\end{abstract}

Keywords: nearshore sandbars; morphodynamics; patterns; process-based modeling

\section{INTRODUCTION}

Nearshore sandbar morphology has been observed to vary in time. Crescentic bars show an alongshore sequence of shoals and channels. In these channels, off-shore directed rip currents generally occur. Under high energy conditions, these shoals have been observed to connect, while rip channels disappear and the bar becomes alongshore uniform (a reset-event). With subsequent lower energy conditions, rip channels reappear and the bar moves shoreward, ultimately welding to the shore through a number of beach states as defined by Wright and Short (1984), a down-state evolution. They based their analysis on the observed behaviour at various Australian beaches. Lippmann and Holman (1990) extended the work by Wright and Short (1984) by distinguishing two additional classes. They defined a sequence of eight beach states, based on four characteristics of the morphology (existence or absence of a bar, dominant bar scaling, longshore variability and trough). They based their analysis on a two year data-set of video images of Duck, North Carolina, USA.

Holman et al. (2006) analysed four years of high resolution (both in time and space) video data from Palm Beach, New South Wales, Australia. Palm Beach is a pocket beach of around $2 \mathrm{~km}$ length, with a single bar, which template is highly variable in time. They used the video-based dissipation patterns as an estimate for the bar location and indicated rip locations on these images. Analysing these rip locations over time allowed an examination of spacings, persistence and location preferences of these rip channels and corresponding hydrodynamic conditions. Their main conclusion was that the length scales could not be matched with concurrent conditions.

Turner et al. (2007) analysed the behaviour of a $2 \mathrm{~km}$ stretch of a long, straight beach at the Goldcoast, Queensland, Australia in a way similar to Holman et al. (2006), using three years of videobased data. Their findings were similar, though the mean rip spacings were slightly different. They also found no tendency for preferred rip locations alongshore following storm reset-events. The alongshore spacing varied similarly. Further, they found no clear relationship between 'the number of rips and prevailing offshore wave conditions, including significant wave height, peak wave period and incident wave power'. Based on these findings they state that 'rips may become rapidly topographically controlled soon after a reset event, and their location is then primarily determined by the evolving nearshore morphology, rather than the hydrodynamic forcing.' Their findings reinforce the need to investigate the role of the antecedent bathymetry in the response to hydrodynamic conditions.

Several efforts have been made to link bar behaviour with either hydrodynamic conditions or bar geometry using both linear stability analyses (LSA) and depth-averaged (2DH) process-based models.

Small alongshore variations in either the bathymetry or the hydrodynamic forcing result in small bathymetrical changes on an initially alongshore uniform profile, which are reinforced by

\footnotetext{
${ }^{1}$ Spatial Ecology, CEME, The Netherlands Institute of Ecology (NIOO-KNAW), P.O. Box 140 Yerseke, 4400 AC, The Netherlands

Formerly at Civil Engineering and Geosciences, Delft University of Technology, P.O.Box 5048 , Delft, 2600 GA, The Netherlands

2 Division of Applied Marine Physics, Rosenstiel School of Marine and Atmospheric Science, University of Miami, 4600 Rickenbacker Causeway, Miami, City, Florida, FL 33149, United States of America

${ }^{3}$ Civil Engineering and Geosciences, Delft University of Technology, P.O.Box 5048, Delft, 2600 GA, The Netherlands
} 
morphological feedback and thus the system evolves toward crescentic patterns (down-state) (e.g. Falqués et al., 1996, 2000; Garnier et al., 2006). Studies of single barred systems based on linear stability analyses showed for example that for obliquely incident waves, the length scales increase with increasing trough area and increasing alongshore current velocities (Deigaard et al., 1999). For shore normal incident waves Damgaard et al. (2002) found that the length scales remained unchanged with increasing wave height, only the growth rate increased, whereas Calvete et al. (2007) found increasing length scales for increasing wave heights. In addition, Calvete et al. (2007) point out that slight variations in the characteristics of cross-shore depth profiles, such as water depth above the crest, rather than the offshore wave conditions can be more important for rip channel spacing. An advantage of 2DH morphodynamic models over linear stability analysis is that they allow for temporal and alongshore variations in crescent lengths and for finite amplitude effects (e.g. Damgaard et al., 2002; Reniers et al., 2004; Smit et al., 2004). This is consistent with Van Enckevort et al. (2004) who observed variations in the field up to a factor 2 in the wavelength and amplitude of individual crescents at any moment in time. The modelling study by Reniers et al. (2004) for example, predicts a variation which agrees with the measurements presented by Holman et al. (2006). Klein and Schuttelaars (2006), who investigated the morphodynamic evolution of double barred beaches for both the linear and nonlinear regime, found that the spacings predicted with the linear stability analysis are only observed during the exponential growth phase of the non-linear experiments. In the dynamic phase, multiple modes span the bed features.

Many modelling efforts concern the response of an initially alongshore uniform bar to wave conditions. However, it has been observed, that sandbar systems in the field generally have a high level of alongshore variability (e.g. Holman et al. 2006). The role of this variability in the sand bar response should therefore also be considered in forecasting the expected morphological response to offshore wave conditions.

Different morphologic patterns affect the way offshore waves will transform when approaching the coast and will thus affect local flows and subsequently, via a feedback mechanism, affect the local morphology and thus the spatial distribution and volume of sand in front of the coast. Different processes have been attributed a role in the formation of the observed templates/patterns (e.g. wave height, wave groups, antecedent morphology). Their formation and evolution is however not yet fully understood. In order to be able to predict the morphological evolution, one needs to understand the roles of various properties of and the state of the system and the role of its forcings.

The current work investigates when the hydrodynamics are the dominant factor in the evolving nearshore patterns and in which cases the pre-existing patterns play a major role. This is approached by analysing a suite of modelling efforts aiming to understand the system's intention when the evolution is not bound by pre-existing morphological variation. In these cases the initial bathymetry was alongshore uniform. The role of the antecedent morphology was tested by varying the initial random perturbation of order $\mathrm{cm}$ and by increasing the level of variability of an initial pattern as well as by investigating the modelled evolution of a hindcasted reset-event. The role of the hydrodynamics is investigated by applying various constant and piece-wise constant conditions as well as observed conditions for a hind-cast case. These methods, as well as the method of analysis, are introduced briefly. This is followed by a description of the model results and the corresponding conclusions to the question: what determines nearshore sandbar response?

\section{METHOD}

We use the results of a suite of depth-averaged process-based morphological modelling efforts to establish the role of alongshore variability on nearshore bar response. The model is a model train consisting of a module for wave transformation (SWAN, refer to Booij et al. 1999), flow formulations and sediment transport. A newly computed bathymetry is then used for the input of the wave transformation resulting in new flow formulations and sediment transport and a new bathymetry. For detailed descriptions of this research version of Delft3D (Lesser et al., 2004) we refer to Smit et al. (2008), Reniers et al. (2004) and Smit (2010).

Computations include constant forcing, piece-wise constant forcing and natural forcing initialized with varying degrees of alongshore bathymetric variability (Table 1). Starting with an initially alongshore uniform bathymetry forced with constant wave conditions resulted in the evolution of crescentic patterns with length scales forced by the hydrodynamic conditions. (Smit et al., 2008) also 
note the role of the cross shore profile in the response to off-shore conditions: depth and volume of the bars. In the case of piece-wise constant forcing it was shown that the adaption of the length scales depends on the bathymetric variability at the moment of transition. This shows the role of the initial variability in the response of the system to changing hydrodynamic conditions (Smit et al., in review). In addition a hindcast was performed of a ten day observed morphological evolution including both up-state and down-state transitions. For a detailed description of the results refer to Smit et al. (in preparation).

\begin{tabular}{|l|l|}
\hline $\begin{array}{l}\text { Table 1. Computational settings: initial bathymetry and type } \\
\text { of forcing conditions }\end{array}$ \\
\hline Initial bathymetry & Forcing condition \\
\hline Alongshore uniform, double bar & Constant conditions \\
Alongshore uniform, double bar & $\begin{array}{l}\text { Piece-wise sequence of } \\
\text { conditions }\end{array}$ \\
Observation-based, single bar & Measured hydrodynamic \\
& conditions \\
\hline
\end{tabular}

\section{Analysing parameters}

The results of the computations were analysed on the evolving length scales, the evolution of the level of morphological variability and the response times of the system. The length scales are a weighted value of the rip channel distances obtained with fourier transformation based on the depth contours. The level of morphological change within an area is defined as the root mean square (RMS [m]) of the differences between the actual depth and the corresponding alongshore mean at each timestep. The response time is the time it takes an initially alongshore uniform bar to respond to the applied constant hydrodynamic forcing. This is defined by the moment at which a certain amount of morphological variability occurs. Refer to Smit et al. (2008) for details of these parameters.

\section{RESULTS}

\section{System's Intention}

The intention of the system is the response the system would show if it would have a constant forcing and no morphologically induced restrictions, other than those due to the initially alongshore uniform profile. It was found that an initially alongshore uniform double barred beach responds distinctly different to different hydrodynamic conditions (Smit et al. (2008)). This leads to the conclusion that a system responds to hydrodynamic conditions and that its response cannot be purely attributed to the systems initial geometry. We used a process-based model to compute the morphological evolution as a response to two weeks of constant wave conditions (Figure 1 illustrates one of these evolutions). The evolution showed alongshore variable distances between rip channels, similar to observations in the field (e.g. Van Enckevort et al., 2004). The weighted length scale as a result of different forcings showed clear trends of increasing length scales for increasing alongshore currents (either due to more oblique waves or due to higher waves) and increasing depths. The length scales ranged from 300-700 $\mathrm{m}$ for the inner bar, and from $600-2000 \mathrm{~m}$ for the outer bar. The length scales are thus a function of both the geometry and the hydrodynamic conditions.

The initial bathymetry was perturbed with a random seed (order $\mathrm{cm}$ ). Analysing computations with 5 different random seedings showed that the different seedings do not alter the mean length scales, but they do alter the location of the rip channels and evolving features. The rip channel location seems therefore to be determined by the antecedent morphology.

The response time of the system was in the order of days and found to depend linearly on the local wave height, the alongshore current, the steepness of the bar and inversely on the active volume of the bar. The results of the model computations agreed with the hypothesis that smaller bar volumes respond more rapid (Van Enckevort et al., 2004). The response time of the system is determined by both the nearshore geometry and the local hydrodynamics. The latter are a function of both the offshore wave conditions, geometry and the nearshore hydrodynamic processes. A nearshore bar system may not always evolve toward new conditions as conditions may not last long enough to allow the system to change (related to the response time of the system). 


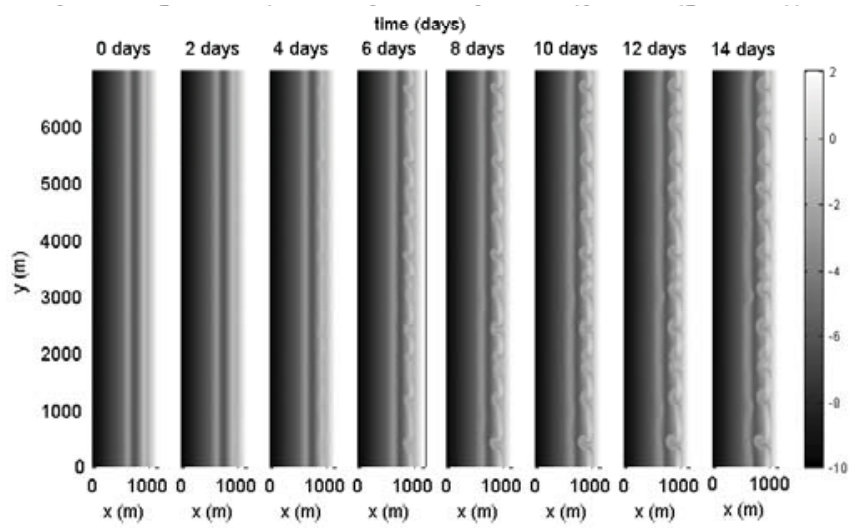

Figure 1. Example of morphological evolution for constant wave conditions and small initial bathymetric variability.

\section{Role of Antecedent Morphology}

To investigate the role of the antecedent morphology, the effect of the initial level of variability in the morphology was tested. Different bathymetries with increasing levels of morphological variability were tested with identical offshore wave forcing. The resulting nearshore hydrodynamic patterns prohibited adaptation toward the new wave conditions of bathymetries with a high level of initial variability, due to the differences in nearshore morphology (e.g. deeper channels). Computations with increasing level of initial morphological variability showed decreasing levels of adaptability when different hydrodynamic conditions forced the system.

A morphology with a deeply imprinted pattern turns out to have a lasting effect on the hydrodynamic patterns, the circulation cells will remain even if offshore wave conditions change significantly and thus the morphology will remain unchanged (at the time scales considered here). If the morphology has more shallow variations, different hydrodynamic conditions may lead to a changed hydrodynamic pattern, allowing the morphology to change (Smit et al., in review).

This shows that, even if the new conditions would remain for an extended period of time, the bathymetry may not adjust toward these new conditions. Whether a system responds to a new condition, turns out not only to depend on the response time, but also on the antecedent morphology.

This may explain why in the work of both Holman et al. (2006) and Turner et al. (2007) observed patterns could not be matched with hydrodynamic conditions for long time periods. There are in fact three reasons. First, in case the level of nearshore variability is high, it prohibits the adaptation to new hydrodynamic conditions. Second, for the post-storm events (in cases that the variability was in fact low enough), the response time and short duration of post-storm conditions may prevent the observed pattern to match the expected pattern (e.g. De Schipper et al., this issue). Third, whether the bathymetrical variability is actually really low enough may not always be completely visible during higher energy events (due to wide band of breaking waves) and the overly calm poststorm conditions when no dissipation patterns are visible. Our findings agree with work by Huntley and Coco (2009), who investigated the behaviour of patterns in small bedforms using abstracted models. They found that the initial state of the seabed has a profound influence on the rate at which the bed responds to new conditions. Also they found that new bedforms can only grow after existing variability has decreased, consistent with the findings of Plant et al. (2006).

\section{Conclusions on Model Performance}

When there is small initial variability, the relative error in this bathymetry may be large. Combining this with the large sensitivity to small variations, it is likely that computations with larger initial variability will result in better matching of the observed evolution.

Users of morphological models should be aware that hydrodynamic calibration is no guarantee for a morphologically correct prediction. A hydrodynamically calibrated model may give highly variable outcomes for morphological computations, depending on the chosen process settings and calibration parameter values. Morphological models should therefore always be calibrated morphologically as well as hydrodynamically. The used model is capable of modelling down-state transitions and one-state upstate transitions to some extent. As reset-events are found to occur infrequently, the model is expected to be capable of hindcasting most observations of several days length. Further, it was found 
that above a certain level of morphological variability, the bar system will not change pattern anymore unless a reset-event occurs. As nearshore bathymetries generally have a reasonable amount of variability, it is expected that downstate evolutions can be modeled and predicted reasonably well, certainly within a period of 3-5 days -and longer for nearshore systems with a large response timewhich generally equals the window of a reasonable level of certainty for wave predictions.

Place and time are crucial, also in morphological modelling. The location of features and their size will affect where new features may emerge (if starting from a low level of variability, order $\mathrm{cm}$ ) or whether the nearshore bar system will remain unchanged (if initial bathymetry has larger level of variability). In the latter case, only extreme conditions will be able to affect the system and result in a changing bathymetry. Time is important in the modelling as the computed morphological evolution needs to occur at the same rate as the observed, otherwise the level of variability (how far features are evolved) will not match the observations and then the model may for example allow the bathymetry to adapt to new conditions, while in the field the features are too deeply imprinted to allow changes. Further in time, the duration of conditions and order of conditions will affect the morphological evolution.

\section{Discussion}

The main research question aimed to explain what determines nearshore sandbar response. From work by others it is known that crescentic patterns initially form due to morphological feedback of small perturbations (either on the morphology or in the wave pattern) and the hydrodynamic forcing (e.g. Falqués et al., 1996, 2000, Garnier et al., 2006). Once patterns are being formed, the remaining questions are why these patterns seem to have certain length scales, certain levels of variability and when and how these patterns change. The role of respectively the hydrodynamics, the geometry and the pre-existing pattern was investigated in the current work. The findings are summarized in Table 2.

\begin{tabular}{|l|l|}
\hline \multicolumn{2}{|l|}{ Table 2. System properties, processes and their effects, hydro = hydrodynamic conditions, } \\
geo = geometry, variability = level of morphological variability. \\
\hline property/parameter/process & affects \\
\hline hydro + geo & length scale and response time \\
perturbation & location of rips \\
variability & level of adaptation \\
\hline
\end{tabular}

Observed nearshore patterns are formed by a combination of the initial bathymetry (the location of features and cross-shore profile: depth of the bar crest and volume of the bar), the level of variability therein (affects the adaptability of the system) and the level of wave energy forcing the system (Figure 2). The performance of the modelling of the evolution of the nearshore morphology is determined by the above, by the accuracy of the morphology and by the quality of the description of the processes. Upto a certain level of morphological variability, the patterns will change in response to the local hydrodynamics and the length scales will adjust accordingly when the conditions prevail long enough. After a certain level of morphological variability has been reached, the occurring length scales will remain, until a reset-event occurs, resulting in a decrease of the variability as the bar becomes alongshore uniform and thus has an infinite length scale.

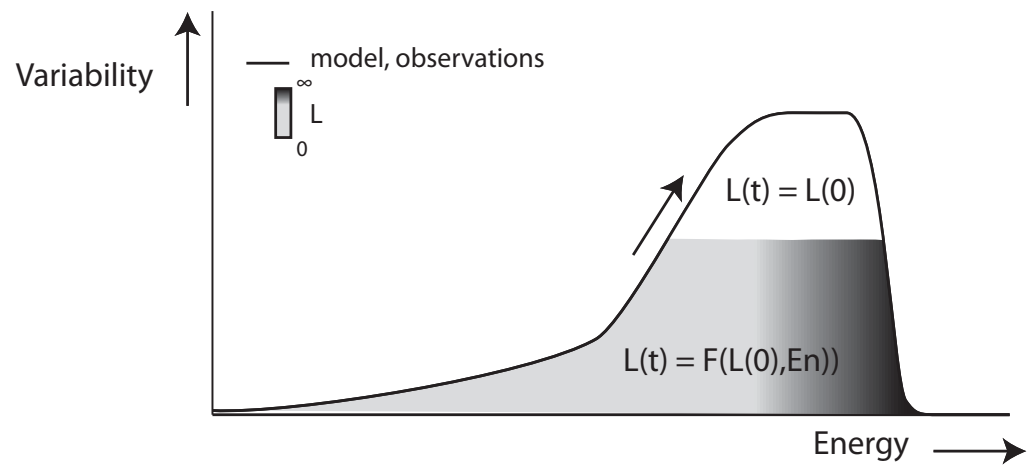

Figure 2. The actual variability of a nearshore bathymetry depends on the initial level of variability and the hydrodynamic conditions. With a high level of variability, no changes will occur (length scale (L) stays constant). If the hydrodynamic energy is very high, it will force the variability to decrease rapidly, leading to a reset-event. 
In general it can be stated that nearshore morphology is sensitive to

- bathymetry (location of perturbations and features and level of variability),

- local conditions,

- $\quad$ prolonged conditions.

\section{CONCLUSIONS}

From the whole suite of modelling efforts, we conclude that sand bar response is determined by the local hydrodynamics as well as the inherited morphological pattern and level of morphological variability therein. With a low level of initial variability in the bathymetry, the hydrodynamic conditions determine the length scales and response times of the system (e.g. Smit et al. 2008). For an increased variability the bathymetry will have deeper evolved rip channels and a more crescentic appearance of the bar crests. In these cases, the hydrodynamic forcing will only affect the existing pattern if the hydrodynamic energy is high, resulting in a removal of alongshore variability and ultimately a reset to an alongshore uniform barred profile.

\section{ACKNOWLEDGEMENTS}

The research was funded by the Dr. Ir. Lely foundation and the ONR-funded Beach Wizard project (N000140510266). AR was funded by NWO under contract DCB 5856. We thank Deltares (formerly known as WL Delft Hydraulics) for providing us with their Delft3D software. We would like to thank CSIRO Marine and Atmospheric Research, Perth, WA, Australia for their hospitality.

\section{REFERENCES}

Booij, N., R.C. Ris, and L.H. Holthuijsen. 1999. A third-generation wave model for coastal regions, part i, model description and validation Journal of Geophysical Research, 104:7649-7666.

Calvete, D., G. Coco, A. Falquès, and N. Dodd. 2007. (un)predictability in rip channel systems, Geophysical Research letters, 34(L05605).

Damgaard, J., N. Dodd, L. Hall, and T. Chasher. 2002. Morphodynamic modelling of rip channel growth, Coastal Engineering, 45((3-4)):199-221.

Deigaard, R., N. Drønen, J. Fredsøe, J., Hjelmager, and M.P. Jørgensen. 1999. A morphological stability analysis for a long straight barred coast, Coastal Engineering, 36:171-195.

De Schipper M.A., R. Ranasinghe, A.J.H.M. Reniers and M.J.F. Stive. 2010. On the initiation of nearshore morphological rhythmicity, Proceedings of International Conference on Coastal Engineering, Shanghai, China.

Falqués, A., A. Montoto, and V. Iranzo. 1996. Bedflow instability of the longshore current, Continental Shelf Research, 16(15):1927-1964.

Falqués, A., G. Coco, and D.A. Huntley. 2000. A mechanism for the generation of wave-driven rhythmic patterns in the surf zone, Journal of Geophysical Research, 105(C10):24,071-24,087.

Garnier, R., D. Calvete, A. Falqués, and M. Caballeria. 2006. Generation and nonlinear evolution of shore oblique/transverse bars, Journal of Fluid Mechanics, 567:327-360.

Holman, R.A., G. Symonds, E.B. Thornton, R. Ranasinghe. 2006. Rip spacing and persistence on an embayed beach, Journal of Geophysical Research, 111(C01006).

Huntley, D.A. and G. Coco. 2009. How do bedforms respond to changing conditions? A study using abstracted models, Proceedings of Coastal Dynamics, Keynote, Tokyo, Japan.

Klein, M.D. and H.M. Schuttelaars. 2006. Morphodynamic evolution of double-barred beaches, Journal of Geophysical Research, 111(C06017).

Lesser, G.R., J.A. Roelvink, J.A.T.M. Van Kester, and G.S. Stelling. 2004. Development and validation of a three- dimensional morphological model, Coastal Engineering, 51(8-9):883-915.

Lippmann, T.C. and R.A. Holman. 1990. The spatial and temporal variability of sand bar morphology, Journal of Geophysical Research, 95(C7), 11575-11590.

Plant, N.G., K.T. Holland, and R.A. Holman. 2006. A dynamical attractor governs beach response to storms, Geophysical Research Letters, 33(L17607).

Reniers, A.J.H.M., J.A. Roelvink, and E.B. Thornton. 2004. Morphodynamic modelling of an embayed beach under wave group forcing, Journal of Geophysical Research, 109(C01030).

Smit, M.W.J., A.J.H.M. Reniers, M.J.F. Stive, and J.A. Roelvink. 2004. Non-linear behaviour of a double nearshore bar system, Proceedings of International Conference on Coastal Engineering, Lisbon, Portugal.

Smit, M.W.J., A.J.H.M. Reniers, B.G. Ruessink, J.A. Roelvink. 2008. The morphological response of a 
nearshore double sandbar system to constant wave forcing, Coastal Engineering, 55(10), 761-770.

Smit, M.W.J., A.J.H.M., Reniers, and M.J.F. Stive. (In Review). Role of morphological variability in the evolution of nearshore sandbars. Coastal Engineering.

Smit, M.W.J., 2010, Formulation and Evolution of Nearshore Sandbar Patterns, PhD Thesis Delft University of Technology.

Smit, M.W.J., A.J.H.M. Reniers, G. Symonds, B.G. Ruessink and M.J.F. Stive. in preparation. Hindcasting up-state and down-state transition at Palm Beach, NSW, Australia, Journal of Geophysical Research.

Turner, I., D. Whyte, B.G. Ruessink, and R. Ranasinghe. 2007. Observations of rip spacing, persistence and mobility at a long, straight coastline, Marine Geology, 236:209-221.

Van Enckevort, I.M.J., B.G. Ruessink, G. Coco, K. Suzuki, I.L. Turner, N.G. Plant, and R.A. Holman. 2004. Observations of nearshore crescentic sandbars, Journal of Geophysical Research, 109(C06028).

Wright, L.D., and A.D. Short. 1984. Morphodynamic variability of surf zones and beaches: a synthesis, Marine Geology, 56, 93-118. 